\title{
DISEÑO PRELIMINAR DE UN ROTOR DE ONDA PARA MOTORES CON MICROTURBINA
}

(1)

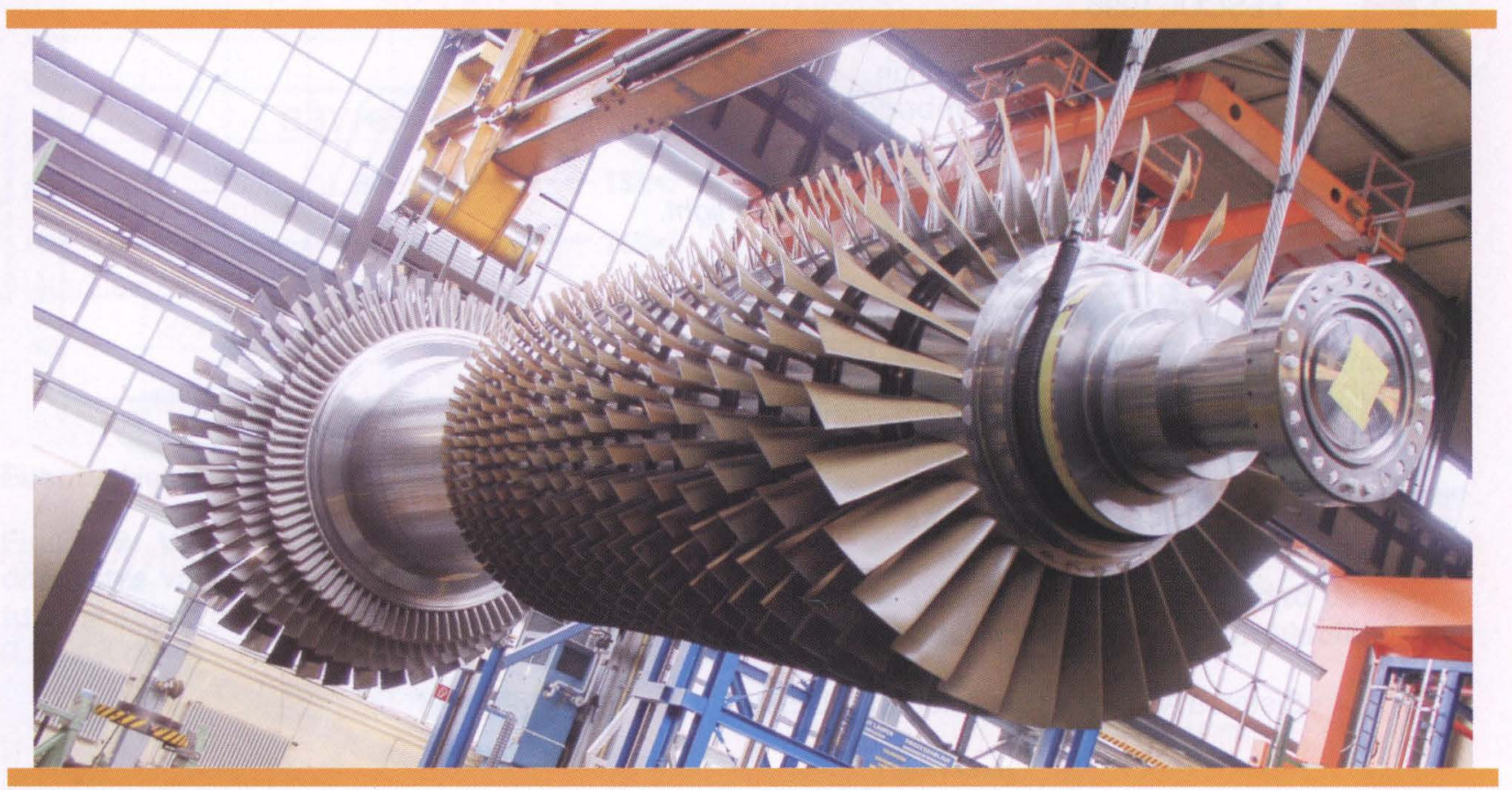

\section{RESUMEN}

Este trabajo presenta un análisis preliminar sobre los beneficios de ia implementación de un rotor de onda en una micro-turbina convencional. Este estudio se enfoca en la consecución de un punto de diseño por medio de cálculos térmicos para así lograr una geometría adecuada, la cual podrá ser evaluada por medio de Dinámica de Flujo Computacional CFD para determinar la viabilidad de la implementación de este dispositivo en un motor con micro-turbina a gas de $30 \mathrm{lb}$, de empuje.

Palabras claves. Termodinámica, cámara de combustión, rotor de onda.

\section{ABSTRACT}

This article shows the benefits of using a wave rotor on small turbo jet engine $(\mu T)$, designed and built at San Buenaventura University [6]. The investigation is focused in a design method that obtains the correct geometry using thermodynamic calculus. CFD (Computational Fluid Dynamics) is used to evaluate the viability in the engine. The equations for the wave rotor preliminary calculus are found through this paper. The input data for the wave rotor design is supported in basic thermodynamic calculus of the engine without the wave rotor.

Key words. Thermodynamic, combuster chamber, wave rotor.

\section{INTRODUCCIÓN}

En este documento se presenta de manera somera el concepto de rotor de onda para motores a reacción pequeños, para uso industrial y académico en algunas asignaturas, de tal manera que se pueda emplear como una solución alterna para la propulsión de aeronaves no tripuladas UAV y el abastecimiento energético en el país generando así una herramienta de estudio en diseños experimentales.

Historia del Rotor de Onda. Los rotores de onda - máquinas de onda de presión, fueron desarrolladas por Brown Boveri y Cia y Jenny hacia los años 40. BBC dio inicio a sus investigaciones para la implementación de un rotor de onda para una planta locomotora con turbina a gas de British Railways[1].

En el periodo de 1956 hasta 1963, fueron realizados algunos trabajos experimentales sobre rotores de onda por la compañía General Electric.

* Grupo de investigación motor combustión GIMOC categoría B colciencias, Universidad de San Buenaventura - Bogotá

** Grupo de investigación en tecnología y desarrollo en logística y seguridad aérea, GITELS registrado en Colciencias, Instituto Militar Aeronáutico-Fuerza Aérea Colombiana 
Fue iniciado un estudio para la reducción del número de etapas del compresor de un motor turbo T-58 GE-06.

Hacia mediados de 1960, Rolls-Royce en cooperación con BBC dieron inicio al desarrollo de rotores de onda intercambiadores de presión como una unidad mejorada en aplicaciones de turbinas a gas.

Entre 1978 y 1985 Mathematical Science Northwest Inc. produjo los diseños preliminares del rotor de onda para una pequeña generación de motores turbofan de cerca de $250 \mathrm{Kg}$ de empuje.

Akbari, Müeller y Piechna iniciaron estudios termodinámicos para evaluar los beneficios del rotor de onda en micro turbinas. La implementación de varios ciclos maximizados de rotores de onda para tener sobre todo eficiencia térmica y aumento hasta de un $34 \%$ para un motor de $30 \mathrm{Kw}$ de potencia, usando un rotor de onda de 4 puertos con relación de compresión de 1.8[14].

\section{DESCRIPCIÓN}

Es un dispositivo de geometría sencilla, que basa su funcionamiento en el empleo de ondas de choque para transferir energía utilizando como medio un fluido, quedando cada uno de sus elementos expuestos tanto al calor como al frío permitiendo así, que los materiales de los componentes del motor no sufran daños debido a las altas temperaturas que se experimentan durante el trabajo de la máquina[2].

El rotor de onda es un elemento que no emplea dispositivos mecánicos para comprimir el fluido, para ello se vale de las ondas de presión generadas en los canales de este, cuya geometría puede ser rectangular, trapezoidal o helicoidal.

\section{Figura 1. Rotor de onda de cuatro puertos}

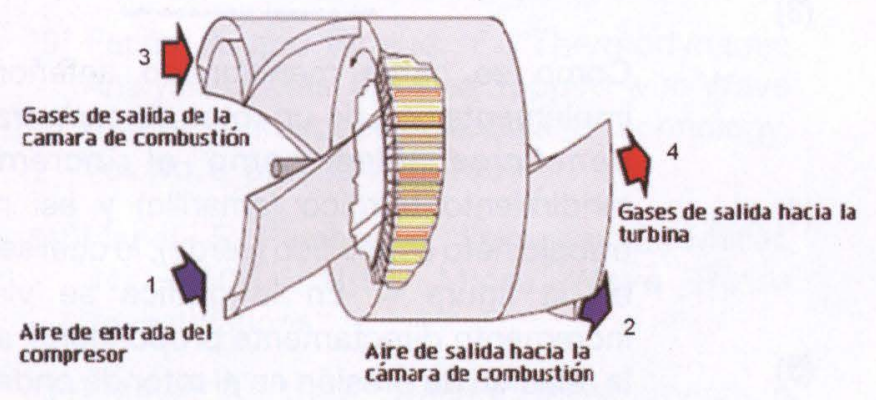

Fuente: Paxson, D., and Lindau, J., 1997, "Numerical Assesment of Four Port through Flow Wave Rotor Cycles with Passages Height Variation", 33rd joint Propulsion Conference and Exhibit, AIAA 97-3142.
Los canales van ubicados alrededor de un cilindro, el cual gira entre dos placas dispuestas en cada extremo[2], en ellas se ubican los puertos, los cuales permiten la entrada y salida de flujo desde y hacia el rotor ver figura 1. La cantidad de estos varía de acuerdo a su utilización, pueden tener desde dos hasta nueve.

Los puertos sirven como medio de control del fluido dentro de los canales. A medida, que el cilindro va girando los canales quedan expuestos a los puertos ubicados en las placas para así dar inicio a los proceso tanto de compresión como de expansión.

Figura 2. Esquema del ciclo de una turbina a gas mejorado con un rotor de onda de cuatro puertos de flujo directo.

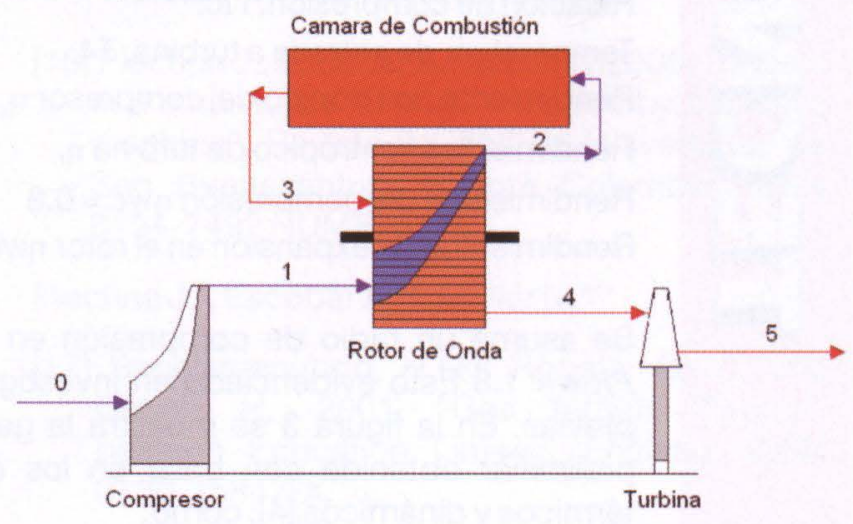

De acuerdo con la trayectoria de flujo en la figura 2, una vez el aire frío descargado del compresor entra al rotor (1), se da inicio al trabajo de compresión dentro del rotor, una vez se ha cumplido el ciclo, este aire precomprimido entra a la cámara de combustión (2), con una mayor presión y temperatura que en el motor base, lo que garantiza una mezcla de combustible-aire enriquecida.

Después de efectuarse el proceso de combustión, los gases calientes, entran al rotor (3), para darse inicio al proceso de expansión. En este momento, tanto el gas caliente como el frío quedan en contacto generándose de esta manera un intercambio de energía; lo que permite elevar la temperatura del aire frío que ha entrado al rotor, para así dar inicio a un nuevo ciclo.

Inmediatamente el gas cumple su proceso de expansión es descargado a la turbina (4). Dependiendo el caso de aplicación, el gas puede entrar a la turbina con la misma temperatura que en el motor base, pero no con la misma presión ya que se ha dado un incremento de esta a través del rotor.

El mayor beneficio de estas máquinas de flujo no estable, es su potencial para generar un aumento de presión mucho mayor que en los dispositivos de flujo estable.




La ganancia obtenida es cercana a un $15 \%$ a $16 \%$ con respecto al motor base, esto debido a la preexpansión en el rotor, lo que asegura un mayor extracción de trabajo del motor. Además, se cuenta con un aumento en el rendimiento térmico y el trabajo específico entre un $20 \%$ y $34 \%$ y esta acompañado de una disminución de SFC que oscila entre el 14\% hasta un $25 \%[3$,$] aproximadamente.$

\section{DISEÑO PRELIMINAR}

Para obtener las condiciones de diseño del rotor se tuvieron en cuenta parámetros del motor base tales como:

Relación de compresión, Пc.

Temperatura de entrada a turbina, $\mathrm{T} 4$.

Rendimiento poli trópico del compresor $\eta_{\mathrm{c}}$

Rendimientos sentrópico de turbina $\eta_{\mathrm{t}}$

Rendimientos del compresión $\eta w c=0.8$

Rendimientos de expansión en el rotor $\eta w e=0.8$.

Se asume un radio de compresión en el rotor $P R w=1.8$ Esto evidenciado en investigaciones previas. En la figura 3 se muestra la geometría preliminar obtenida con base en los cálculos térmicos y dinámicos [4], como:

Trabajo de compresión y expansión dentro del rotor

$W w c=\operatorname{mairCpair}(T t 2-T t 1)=\frac{\text { mairCpairTt } 1}{\eta w c}\left(P R w^{\frac{\text { qair }-1}{\text { Yair }}}-1\right)$

$W w e=($ mair $+m f) \operatorname{Cpgas}(T t 3-T t 4)=($ mair $+m f) \operatorname{Cpgas} \eta w e T t 3 \times\left[1-\left(\frac{P O}{\Pi c o m b P R w}\right)^{\frac{\operatorname{rgas}-1}{\lambda g a s}}\right]$

Trabajo neto específico producido por el motor:

$$
w=W t-W c
$$

Rendimiento térmico:

$$
\eta=\frac{w}{q}
$$

Empuje específico:



$$
S T=(1+f) U 6-U a+a_{0}\left[(1+f) \frac{\operatorname{Rgas}}{R} \frac{\frac{T t 6}{T t 0}}{\frac{U 6}{a_{0}}} \frac{1-\frac{P o}{P 6}}{\text { rair }}\right]
$$

$Y$ finalmente el consumo específico de combustible que es calculado por:

$S F C=\frac{f}{S T}$

Figura 3. Diseño preliminar de un rotor de onda de cuatro puertos

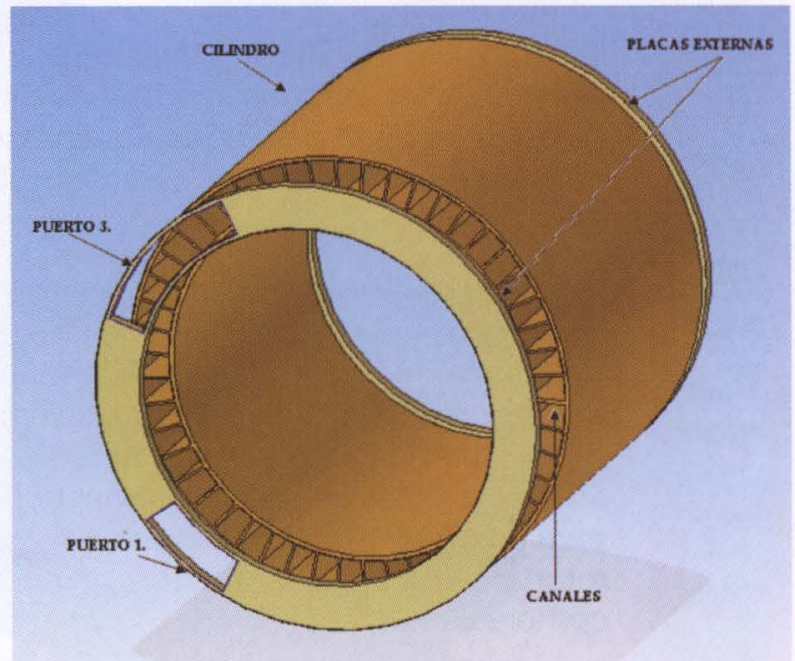

Así mismo, se puede predecir una maximización en el desempeño del motor gracias a la utilización del rotor de onda. Esto se puede ver en la siguiente figura.

Figura 4. Eficiencia térmica y trabajo específico versus radio de presión del rotor de onda

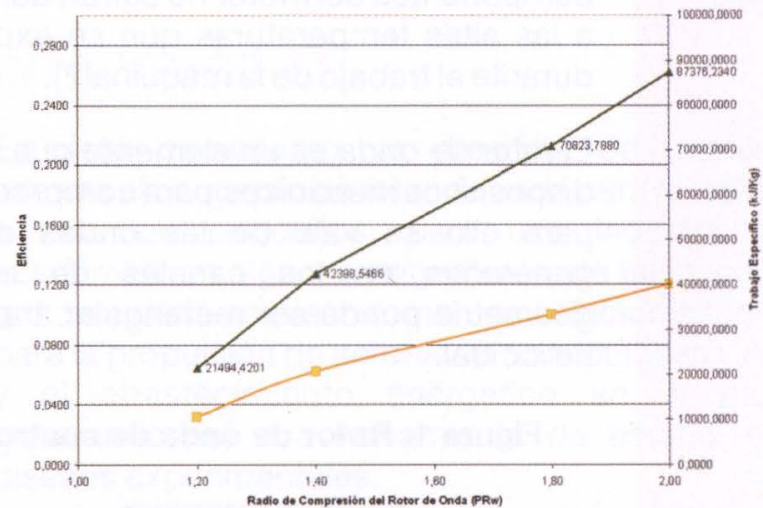

Como se había mencionado anteriormente, la implementación de un rotor de onda trae consigo beneficios tales como el incremento del rendimiento térmico (amarillo) y así mismo del trabajo neto específico (verde), lo cual se puede ver en la figura 4. En la gráfica se visualiza un incremento directamente proporcional al aumento la relación de presión en el rotor de onda. Hasta el momento se ha concebido una relación de presión óptima en el rotor la equivalente a 1.8, con la cual la eficiencia térmica podría tener una ganancia alrededor del $20 \%$ y una disminución de SFC de $20 \%$ aproximadamente. 
REFERENCIAS

[1] Akbari, P., Müller, N., 2003, "Performance Investigation of Small Gas Turbine Engines Topped with Wave Rotors," AIAA-Paper 2003-4414.

[2] Akbari, P., Nalim, R., Müller, N., "Performance Enhancement of Microturbine Engines Topped With Wave Rotors", Journal of Engineering for Gas Turbines and Power, 128, pp 190-202.

[3] Akbari, P., Mueller, N., 2003, "Performance Improvement of Small Gas Turbines Through Use of Wave Rotor Topping Cycles, "2003 International ASME/IGTI Turbo Exposition, ASME Paper GT2003-38772.

[4] Akbari, P., Mueller, N., 2003, "Preliminary Design Procedure for Gas Turbine Topping Reverse-Flow Wave Rotors, "2003 International Gas Turbine Congress, ASME Paper IGTC03-FR-301

[5] Akbari, P., Mueller, N., 2003, "Gas Dynamic Design Analyses of Charging Zone for Reverse- Flow Pressure Wave Superchargers, "2003 ASME Spring Technical Conference, ASME Paper ICES2003-690.

[6] Escobar A., Metodología de diseño para turborreactores, Universidad de San Buenaventura, Bogotá, D. C.

[7] Fatsis, A. and Ribaud, Y., 1997, "Numerical Analysis of the Unsteady Flow Inside Wave Rotors Applied to Air Breathing Engines", 13 International Symposium on Airbreathing Engines.

[8] Fatsis, A. Lafond and Ribaud, Y., 1998, "Preliminary Analysis of the Flow Inside a Three-Port Wave Rotor by Means of a Numerical Model", Aerospace Science and Technology, Vol. 2, No 5, pp.289-300.

[9] Fatsis, A. and Ribaud, Y., "Thermodynamic Analysis of Gas Turbines Topped with Wave Rotors," Aerospace Science and Technology, Vol. No. 5, pp.293-299, 1999

[10] lancu, F., Piechna, J., Dempsey, E., Müller, N., 2005, "Ultra Micro Wave Rotor Investigations".

[11] Meyer, A., 1947, "Recent Developments in Gas Turbines," Journal of Mechanical Engineering, 69, No.4, pp. 273-277.

[12] Okamoto K., Nagashima T., 2003, „A Simple Numerical Approach of Micro Wave Rotor Gasdynamic Design", 16th International
Symposium on Airbreathing Engines, Paper ISABE-2003-1213.

[13] Paxson, D., and Lindau, J., 1997, "Numerical Assesment of Four Port through Flow Wave Rotor Cycles with Passages Height Variation", 33rd joint Propulsion Conference and Exhibit, AIAA 97-3142.

[14] Piechna J., Akbari P., lancu F., and Müller N., 2004, Radial-flow wave rotor concepts, unconventional designs and applications, IMECE2004-59022, Anaheim 2004

[15] Piechna J., 2005, "Wave Machines, Models and Numerical Simulation", Oficyna Wydawnicza Politechniki Warszawskiej, Warszawa 2005, ISSN 0137-2335

[16] Piechna J., Escobar A., Müller N.2006, "project of a small turbo jet engine topped by the wave machine", Revista INGENIUM Universidad de San Buenaventura Bogotá Colombia, ISSN 0124-7492 NUMERO 16

Piechna J. ${ }^{*}$ Escobar A. ${ }^{* \star}$, Müller N. ${ }^{\star \star \star}$

[17] Saravanamuttoo, H.I.H; Rogers, G.F.C, and Cohen, H., 2001, "Gas Turbine Theory", pearson Education Limited, England, ISBN 0130-15847-X.

[18] Snyder, P. H., Fish, R. E., 1996, "Assessment of a Wave Rotor Topped Demonstrator Gas Turbine Engine Concept,"ASME Paper 96-GT-41.

[19] Spring, P., Piechna J., and Onder, C., 2004, Modeling and validation of a pressure-wave supercharger using a finite difference method, IMECE2004-59533, Anaheim 2004

[20] Taussig, R. T., 1984, „Wave Rotor Turbofan Engines for Aircraft", Mechanical Engineering, 106 , No. 11 , pp. $60-68$.

[21] Taussig, R. T., Hertzberg, A., 1984, „Wave Rotors for Turbomachinery,“, Winter Annual Meeting of the ASME, edited by Sladky, J. F., Machinery for Direct Fluid-Fluid Energy Exchange, AD-07, pp. 1-7.

[22] Welch, G.E., Jones, S. M. and Paxson, D. E., 1997, "Wave Rotor-Enhanced Gas Turbine Engines", Journal of Engineering for Gas Turbines and Power, 119, No. 2, pp 469-477.

[23] Wilson, J., Paxson, D. E., 1993, “Jet Engine Performance Enhancement Through Use of a Wave-Rotor Topping Cycle," NASA TM-4486 